\title{
Binary Diffusion Coefficients of the Liquid Organic Hydrogen
}

\section{Carrier System Dibenzyltoluene/Perhydrodibenzyltoluene}

\author{
Andreas Heller, ${ }^{a}$ Michael H. Rausch, ${ }^{a, b}$ Peter S. Schulz, \\ Peter Wasserscheid, ${ }^{\mathrm{c}, \mathrm{d}}$ and Andreas P. Fröba ${ }^{*, a, b}$
}

${ }^{a}$ Erlangen Graduate School in Advanced Optical Technologies (SAOT), University of ErlangenNuremberg, Paul-Gordan-Straße 6, D-91052 Erlangen, Germany

${ }^{\mathrm{b}}$ Department of Chemical and Biological Engineering, Institute of Engineering Thermodynamics (LTT), University of Erlangen-Nuremberg, Am Weichselgarten 8, D-91058 Erlangen, Germany

${ }^{\mathrm{c}}$ Department of Chemical and Biological Engineering, Institute of Chemical Reaction Engineering, University of Erlangen-Nuremberg, Egerlandstraße 3, D-91058 Erlangen, Germany

${ }^{\mathrm{d}}$ Helmholtz-Institute Erlangen-Nuremberg for Renewable Energies, IEK-11, Forschungszentrum Jülich, Nägelsbachstraße 59, D-91058 Erlangen, Germany

* Corresponding author; E-mail: andreas.p.froeba@fau.de. Tel.: +49-9131-85-29789 
Table S1. Refractive Index $n_{D}$ and Refractive Index Difference $n_{F}-n_{C}$ for the Investigated Mixture Compositions as a Function of Temperature $T$ at $0.1 \mathrm{MPa}^{a}$

\begin{tabular}{clcccc}
$T$ & $n_{\mathrm{D}}$ & $n_{\mathrm{F}}-n_{\mathrm{C}}$ & $T$ & $n_{\mathrm{D}}$ & $n_{\mathrm{F}}-n_{\mathrm{C}}$ \\
\hline $\mathrm{K}$ & - & - & $\mathrm{K}$ & - & - \\
& $x_{\mathrm{LOHC}}=0$ & & & $x_{\mathrm{LOHC}}=0.10$ & \\
293.7 & 1.4998 & 0.010 & 297.2 & 1.5069 & 0.011 \\
313.2 & 1.4923 & 0.010 & 313.2 & 1.5011 & 0.011 \\
333.2 & 1.4859 & 0.011 & 332.7 & 1.4933 & 0.011 \\
& & & 344.2 & 1.4888 & 0.011 \\
& $x_{\mathrm{LOHC}}=0.25$ & & & $x_{\mathrm{LOHC}}=0.50$ & \\
294.2 & 1.5216 & 0.013 & 293.7 & 1.5465 & 0.016 \\
313.2 & 1.5139 & 0.012 & 312.7 & 1.5392 & 0.015 \\
331.7 & 1.5065 & 0.013 & 332.2 & 1.5310 & 0.015 \\
347.2 & 1.5000 & 0.012 & 344.2 & 1.5261 & 0.015 \\
& $x_{\mathrm{LOHC}}=0.75$ & & & $x_{\mathrm{LOHC}}=0.90$ & \\
294.2 & 1.5726 & 0.019 & 294.7 & 1.5918 & 0.021 \\
313.7 & 1.5645 & 0.019 & 313.2 & 1.5832 & 0.020 \\
332.2 & 1.5573 & 0.019 & 330.2 & 1.5766 & 0.020 \\
344.7 & 1.5520 & 0.019 & 346.7 & 1.5704 & 0.020 \\
& $x_{\mathrm{LOHC}}=1$ & & & & \\
293.7 & 1.6051 & 0.022 & & & \\
314.2 & 1.5968 & 0.022 & & & \\
331.2 & 1.5893 & 0.021 & & &
\end{tabular}

${ }^{a}$ The combined expanded uncertainties $U_{\mathrm{c}}$ are $U_{\mathrm{c}}(T)=0.5 \mathrm{~K}$ and $U_{\mathrm{c}}(p)=3 \mathrm{kPa}$ (level of confidence = $0.95)$. 


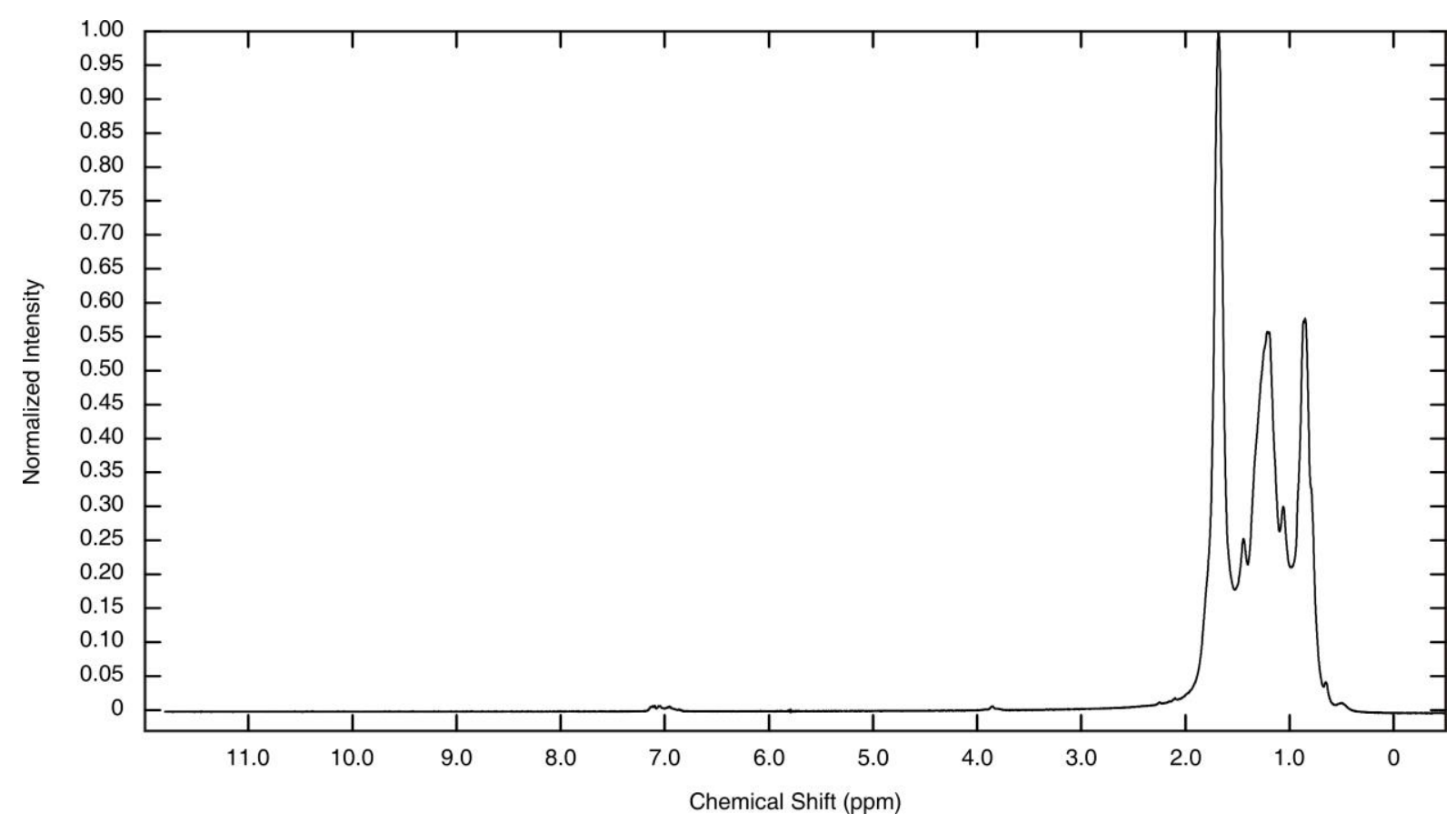

Figure S1. ${ }^{1} \mathrm{H}-\mathrm{NMR}$ spectrum without solvent of $\mathrm{H}_{18}$-LOHC directly after hydrogenation.

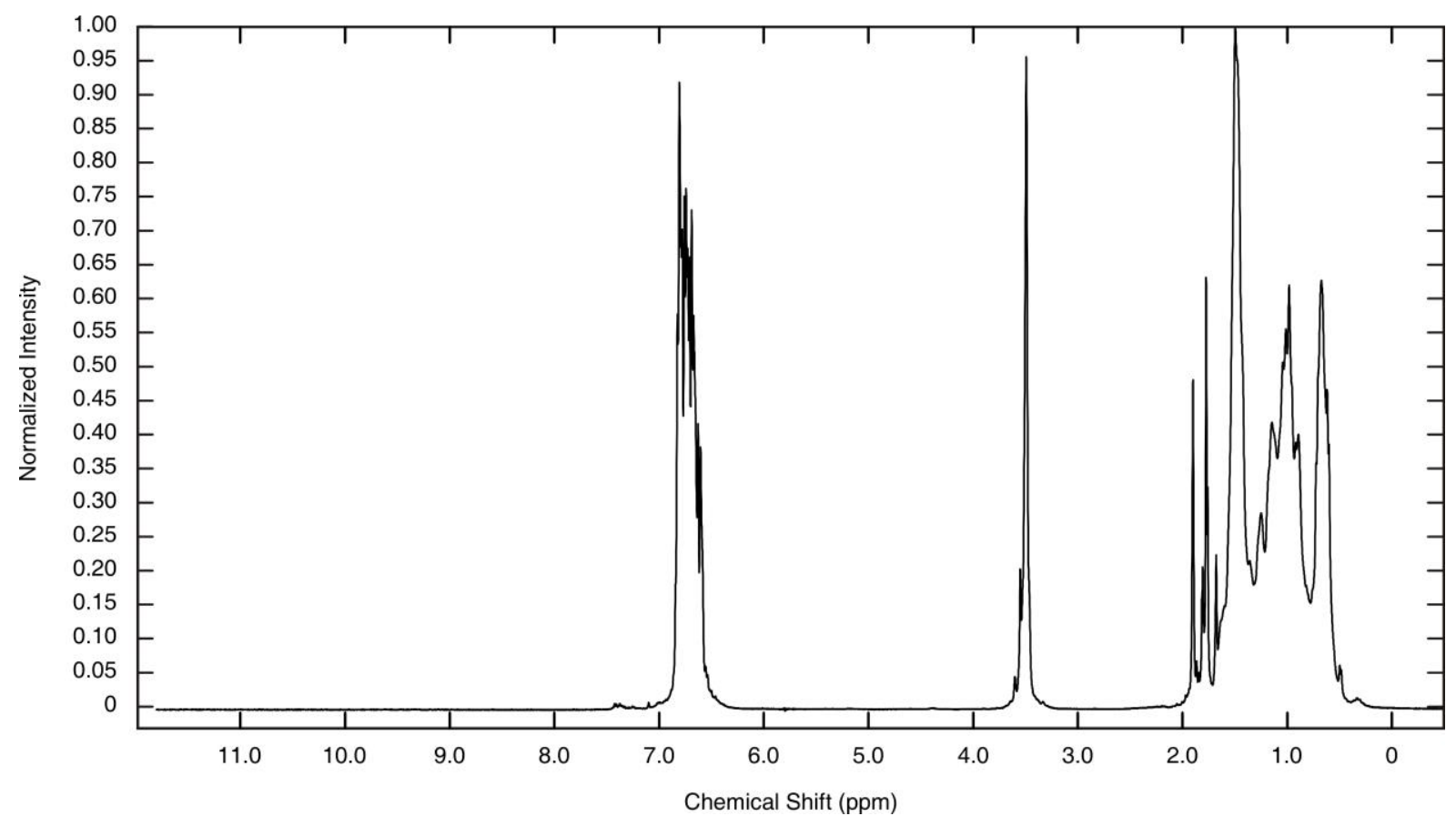

Figure S2. ${ }^{1} \mathrm{H}-\mathrm{NMR}$ spectrum without solvent of $x_{\mathrm{LOHC}}=0.5$ after measurements were performed starting from (352 to 493) K. 